\title{
Evaluación de proceso del programa escolar de prevención del consumo de cánnabis "xkpts.com" en adolescentes de Barcelona en 2006
}

\section{Process evaluation of the school-based cannabis use prevention program "xkpts.com" in adolescents from Barcelona in 2006}

\author{
Francesca Sánchez-Martínez ${ }^{*}$,*****; Carles Ariza

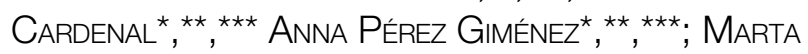 \\ DiéGuez FerRer *; Maria José LóPez MedinA ${ }^{\star},{ }^{* \star},{ }^{* \star *}$; \\ MAnel Nebot AdelL ${ }^{\star * \star},{ }^{* \star *},{ }^{* \star \star \star}$
}

* Servicio de Evaluación y Métodos de Intervención de la Agencia de Salud Pública de Barcelona.

${ }^{* \star}$ CIBER de Epidemiología y Salud Pública CIBERESP.

*** Institut d'Investigació Biomèdica (IIB Sant Pau).

${ }^{* \star \star \star}$ Departament de Ciències Experimentals i de la Salut, Universitat Pompeu Fabra.

\section{RESUMEN}

Introducción: Con frecuencia los efectos positivos observados de las intervenciones preventivas escolares son menores a los esperados, habitualmente por una implementación inadecuada.

Objetivos: Describir la evaluación de proceso del programa de prevención del consumo de cánnabis en escolares (xkpts.com), medir la satisfacción de los profesores con el programa y definir los parámetros cuantitativos y cualitativos para valorar la exhaustividad de la intervención.

Metodología: Estudio descriptivo transversal. Muestra: 117 aulas de 39 escuelas de $3^{\circ}$ ESO (2812 escolares). La información se recogió mediante fichas de evaluación cumplimentadas por el profesorado y las familias. Se realizó un análisis descriptivo de la implementación de la intervención en las aulas y de la participación de las familias.

Resultados: Tasa de respuesta de los profesores del 65\%. La discusión fue la técnica más aplicada (100\% de las aulas) y el DVD el recurso más utilizado $(93,4 \%)$. El entrenamiento de habilidades se aplicó en el 72,4\% de aulas. El 59,2\% de aulas realizó una intervención aceptable (mínimo 8 de 16 actividades preventivas) frente al 38,2\% de aulas con intervención cualificada (mínimo 1 actividad por cada sesión general y 2 por cada especifica). La puntuación media de la valoración del programa fue 7,4 y el $81,6 \%$ del profesorado volvería a aplicarlo. La tasa de participación de las familias fue del 28,3\% (796).

Conclusiones: En dos de cada tres aulas la intervención fue aceptable y cuatro de cada diez cumplieron estrictamente el protocolo. Una mayor participación de las familias se corresponde con las escuelas con una mejor implementación de la intervención.

Palabras clave: Evaluación de proceso. Prevención. Escolar. Cánnabis. Adolescentes
Enviar correspondencia a:

Francesca Sánchez Martínez.

Servei d'Avaluació i Mètodes d'Intervenció. Agencia de Salud Pública de Barcelona. Plaça Lesseps, 1. 08023 Barcelona, España.

Teléfono: 932027746

Correo electrónico: fsanchez@aspb.cat

\section{ABSTRACT}

Background: The observed positive effects of school-based prevention programs are frequently lower than what was expected, usually due to inadequate implementation.

Aims: To describe the process evaluation of a cannabis use school-based prevention program (xkpts.com), to measure teachers' satisfaction with the program and to define quantitative and qualitative parameters for assessing the exhaustiveness of the intervention.

Methods: Cross-sectional descriptive study. Sample: 117 9th-grade classes in 39 schools (2812 students). The information was gathered by means of a self-report questionnaire for teachers and families. A descriptive analysis was made of the implementation of the intervention in the classrooms and of the families' participation.

Results: Teachers' response rate was 65\%. Discussion was the method most widely applied (100\% of the classrooms) and DVD was the most widely used material (93.4\%). Skills training was applied in $72.4 \%$ of the classrooms. In $59.2 \%$ of the classrooms there was an acceptable intervention (at least 8 of the 16 preventive activities), while in 38.2\% there was a qualified intervention (at least 1 activity for each general lesson and 2 for each specific one). Mean score given to the program by teachers was 7.4, and $81.6 \%$ of them reported their intention to apply it again. Participation rate of the students' families was $28.3 \%$ (796).

Conclusions: The intervention was acceptable in two out of three classrooms, while in four out of ten the protocol was applied strictly. Greater participation of the students' families corresponded to those schools in which the intervention was better implemented.

Key words: Process evaluation. Prevention. School-based. Cannabis. Adolescents. 


\section{INTRODUCCIÓN}

$\mathrm{N}$ umerosos estudios han demostrado la efectividad de los programas escolares de prevención que abordan los problemas relacionados con el comportamiento 0 hábitos de consumo de sustancias entre los jóvenes ${ }^{1-4}$. Sin embargo, los efectos positivos conseguidos por estas intervenciones decaen habitualmente en los años sucesivos a la implementación de la intervención ${ }^{5-9}$. Además, con frecuencia el efecto observado tras la aplicación del programa es inferior al esperado. Entre las posibles causas de esta situación se ha sugerido la implementación inadecuada, incluyendo la menor intensidad o duración de las sesiones, unas expectativas previas inapropiadas, limitaciones en el diseño y evaluación del programa, sesgos asociados a la pérdida de los alumnos de mayor riesgo, aplicación del programa en una edad inapropiada o el uso de mensajes inadecuados ${ }^{10}$.

Existen diversos métodos para valorar la implementación basados en variables e indicadores del desarrollo de la intervención en el aula. Aportan información sobre las estrategias, recursos y componentes del programa que se ha aplicado, el tiempo dedicado, el número de actividades preventivas realizadas, la aceptación y satisfacción con el programa así como el grado de seguimiento del diseño inicial. Se han definido dos dimensiones de la implementación: cuantitativa y cualitativa ${ }^{11}$. La dimensión cuantitativa, también conocida como cumplimiento o dosis, aporta información sobre qué cantidad de la intervención ha sido aplicada y normalmente se expresa como el porcentaje del total del contenido que ha sido efectivamente implementado (ex. el porcentaje de sesiones realizadas). Por otro lado, la dimensión cualitativa o fidelidad de implementación ha sido descrita como el grado en que los profesores y otros profesionales que aplican los programas los implementan tal y como señala su diseño y evaluación-piloto ${ }^{11,12}$.

El cuestionario auto contestado por parte del profesorado y la entrevista post-implementación son métodos válidos para medir el cumplimiento mientras que para valorar la fidelidad suele utilizarse la observación de las sesiones (fidelidad observada o fidelidad objetiva), aunque en ocasiones ésta también puede ser valorada a través de los cuestionarios a los participantes (fidelidad autodeclarada o fidelidad subjetiva). A modo de resumen, en la Tabla 1 se muestran los diversos métodos de medición de la implementación más reconocidos en la literatura ${ }^{11}$.

En los últimos años un elevado número de estudios han señalado la importancia de evaluar la fidelidad de la implementación de las intervenciones preventivas escolares ${ }^{13-18}$. Su evaluación nos puede conducir a un mejor entendimiento del éxito o fracaso de un programa ya que la obtención de los resultados esperados está estrechamente vinculada a la intensidad y cualidad de la implementación ${ }^{15,19}$. Una intervención con un buen diseño metodológico puede tener un impacto atenuado si en su aplicación se produce una inadecuada implementación. De igual modo, una pobre fidelidad de implementación dificulta conocer qué componentes contribuyen al éxito de un programa, en caso de conseguir resultados positivos ${ }^{20}$. No obstante, son escasos los programas preventivos que realizan una evaluación de proceso tras su aplicación $y$, cuando se hace, generalmente carecen del rigor necesario para garantizar la correcta aplicación de la intervención en el aula.

Por otro lado, en las intervenciones preventivas escolares cada vez cobra más importancia el componente familiar, ya que los padres juegan un papel muy importante en la educación para la salud de los hijos ${ }^{21}$. Es muy importante que la escuela se ocupe de que los padres estén capacitados para ejercer un papel específico en la educación sobre alcohol y drogas, facilitándoles la formación necesaria para mejorar su capacidad educativa (habilidades de comunicación, conocimiento de los hijos, establecimiento de normas, etc.) ${ }^{22}$. Proporcionar apoyo a las familias se considera una estrategia clave en el abordaje de diversos problemas sociales, tales como la delincuencia o el consumo de drogas ${ }^{23}$, ya que los adolescentes cuyos padres se involucran en su educación tienen menor probabilidad de desarrollar conductas problemáticas ${ }^{24}$. Sin embargo, la tasa de participación de las familias en los programas de prevención del consumo de drogas es, con frecuencia, muy baja $a^{25,26}$.

El objetivo de este estudio es describir la evaluación de proceso del programa de prevención del consumo de cánnabis "xkpts.com"27, iniciativa innovadora en nuestro contexto que aborda la problemática del consumo de esta sustancia entre los jóvenes de nuestro entorno. Así pues, en este estudio se describen los componentes de la intervención, la percepción del profesorado sobre el funcionamiento de las sesiones en el aula, su satisfacción con el programa y su intención de volver a aplicarlo en un futuro, así como se definen los parámetros cuantitativos y cualitativos que permiten valorar la exhaustividad de la intervención. Asimismo, se describe la participación de las familias en la intervención.

\section{MÉTODOS}

\section{Diseño del estudio y selección de la muestra}

El diseño del estudio es descriptivo transversal y forma parte de un estudio más amplio de evaluación de la efectividad del programa de prevención del consumo de cánnabis "xkpts.com" en alumnos de $3^{\circ}$ de Educación Secundaria Obligatoria (ESO) de la ciudad de Barcelona.

Para el estudio de evaluación de la efectividad se seleccionaron 39 escuelas como parte del grupo de intervención y 47 como parte del grupo de comparación. Las escuelas incluidas en este estudio habían sido asignadas aleatoriamente a ambos grupos a partir de un procedimiento de muestreo estratificado según titularidad de la escuela (pública / privada / concertada) y nivel socioeconómico del barrio en el que se ubicaba la escuela. Se tuvo en cuenta que esta distribución fuese proporcional a la de la ciudad de Barcelona, tanto en cuanto a la titularidad de la escuela (69,2\% concertada o privada y $30 \%$ pública) como al nivel socioeconómico del barrio (41,1\% bajo, $30,8 \%$ medio 
Tabla 1. Métodos de medición de la implementación de una intervención en el aula y dimensión de la aplicación del programa que permiten evaluar

\begin{tabular}{|c|c|c|}
\hline Métodos de medición de la implementación & Dimensión de la implementación & ¿Qué permite evaluar? \\
\hline Cuestionario autocontestado por el profesorado & \multirow{2}{*}{$\begin{array}{l}\text { - Cumplimiento } \\
\text { - Fidelidad (autodeclarada) }\end{array}$} & $\begin{array}{l}\text { - Si el profesorado ha realizado cada una de las actividades contenidas en } \\
\text { las distintas sesiones (cumplimiento) }\end{array}$ \\
\hline Entrevista post-implementación al profesorado & & $\begin{array}{l}\text { - En qué medida el profesorado declara que ha completado cada una de } \\
\text { las actividades principales, de acuerdo con las instrucciones propias } \\
\text { del programa (fidelidad autodeclarada) }\end{array}$ \\
\hline \multirow[b]{2}{*}{ Observación no participante en el aula } & \multirow{2}{*}{$\begin{array}{l}\text { - Fidelidad (objetiva) } \\
\text { - Compenetración entre profesorado y } \\
\text { alumnado a partir de la observación } \\
\text { (Rapport) }\end{array}$} & $\begin{array}{l}\text { - Si el profesorado implementa cada uno de los procedimientos con- } \\
\text { tenidos en las sesiones de acuerdo con la intensidad y distribución } \\
\text { presentes en el protocolo del programa (fidelidad objetiva) }\end{array}$ \\
\hline & & $\begin{array}{l}\text { - Interacción entre el profesorado y el alumnado: } \\
\text { - capacidad del profesorado para involucrar al alumnado en las } \\
\text { actividades y discusión } \\
\text { - rendimiento del profesorado en el desarrollo de determinadas } \\
\text { estrategias } \\
\text { - Receptividad del alumnado al contenido de la intervención. (Rapport) }\end{array}$ \\
\hline
\end{tabular}

Elaboración propia a partir de Resnicow K, Davis M, Smith M, Lazarus-Yaroch A, Baranowski T, Baranowski J, et al. How best to measure implementation of school health curricula: a comparison of three measures. Health Educ Res 1998;13(2):239-250.

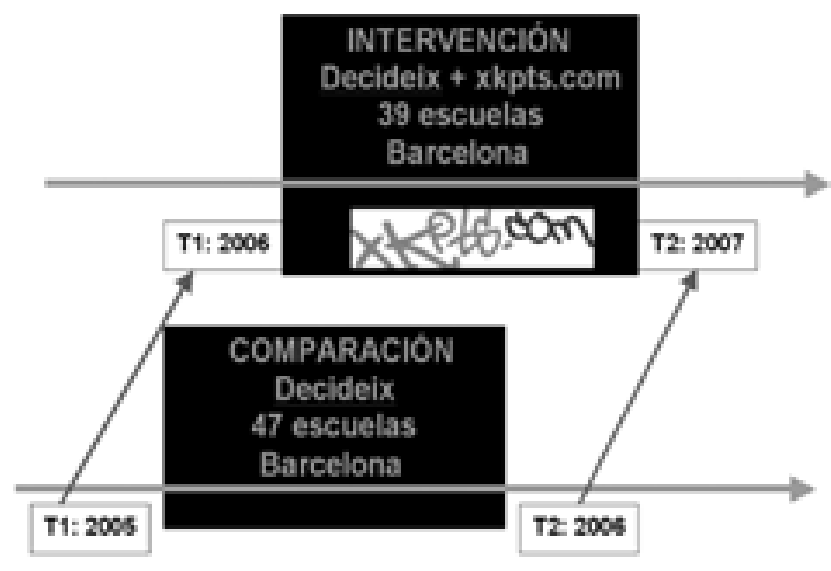

Figura 1. Secuencia temporal del estudio de evaluación de la efectividad del programa de prevención de consumo de cánnabis "xkpts.com" en alumnos de $3^{\circ}$ de ESO de la ciudad de Barcelona

y $28,1 \%$ alto). Como criterio de inclusión, las escuelas de ambos grupos debian expresar su conformidad con el estudio y haber aplicado previamente el programa de prevención de drogodependencias "Decideix!"28, pre-existente en la ciudad de Barcelona, con el fin de garantizar que todas las escuelas participantes en el estudio partían de un mismo nivel basal de prevención. Este programa pretende que el joven aprenda a decidir libremente sobre su consumo, asumiendo las consecuencias de su decisión. Consta de cuatro sesiones, que se plantean mediante cuatro historietas que presentan situaciones que permiten trabaja los conceptos básicos sobre las drogas, el inicio en el consumo de una droga, saber decir no cuando no se quiere consumir y saber actuar en caso de abuso.

Se encuestó al alumnado participante de ambos grupos en dos momentos temporales (T1 y T2) separados por 15 meses, en el caso del grupo intervención antes y después de aplicar el programa. El grupo comparación fue histórico, ya que se encuestó un año antes que el grupo intervención con el fin de evitar la contaminación entre grupos (ver Figura 1).

Los datos que aquí se presentan corresponden a la evaluación del proceso de la aplicación del programa "xkpts. com" en las 39 escuelas que formaban parte del grupo de intervención y que se llevó a cabo durante el tercer trimestre escolar de 2006 (marzo-junio). La muestra estaba formada por 117 aulas de $3^{\circ}$ de ESO, con un total de 2812 escolares.

\section{Descripción de la intervención:}

El "xkpts.com" es un programa de prevención del consumo de cánnabis dirigido a adolescentes de 14 a 16 años. El nombre "xkpts.com" corresponde al juego de palabras resultante del significado de "peta" en el argot juvenil, porro en catalán, y del verbo "petar", que en catalán significa explotar (la pronunciación es "per_que_petas_punt_com"). Este programa pretende aumentar los conocimientos sobre los efectos y las consecuencias del consumo de cánnabis, identificar la presión social para consumir y promover las habilidades para rechazar su consumo. La intervención 
consta de un total de 16 actividades preventivas enmarcadas en cuatro sesiones. En cada sesión se incluyen unas actividades principales, de obligada aplicación, y otras opcionales, que refuerzan el efecto preventivo de las actividades principales. Dos de las sesiones se centran en la toma de decisiones en un marco general de prevención de drogodependencias (sesiones 1 y 4) y las otras dos en la prevención específica del consumo de cánnabis (sesiones 2 y 3 ). La sesión 2 se centra en la presión de grupo mientras que en la sesión 3 se entrenan habilidades de comunicación y de rechazo en situaciones especificas de oferta de cánnabis.

En la Tabla 2 se describen las actividades preventivas comprendidas en cada una de las cuatro sesiones así como las técnicas que se recomienda aplicar y los recursos a utilizar en cada una de ellas según el protocolo del programa. Los recursos utilizados en las sesiones 1 y 4 son unas historietas de lectura individual del programa "Decideix", en las que se representa una situación relacionada con el tema a tratar y se incluyen unas sugerencias para orientar la discusión posterior, así como una breve exposición de informaciones objetivas. En la sesión 2 se utiliza el recurso central del programa, un $D D^{27}$ que incluye una ficción que pretende incitar a los jóvenes a participar en la discusión posterior. Con el fin de facilitar el debate, el DVD incluye una videoguía en la que se proponen seis temas a debatir.

El programa aborda la problemática del consumo de cánnabis desde tres ámbitos clave: escolar, familiar e individual (extraescolar). La intervención incorpora dos recursos de apoyo a la intervención escolar: una guía destinada a las familias de los adolescentes ${ }^{29}$ y la página web del programa www.xkpts.com. La guía contiene información sobre el cánnabis, razones del consumo, consecuencias, posibles respuestas a preguntas comprometidas, cómo actuar ante el consumo de los hijos y recursos de ayuda con el fin de facilitar el diálogo sobre el tema en el hogar. Mediante la página web los jóvenes pueden ampliar sus conocimientos sobre el tema, buscar recursos y resolver dudas.

Tabla 2. Componentes de la intervención de prevención de consumo de cánnabis en adolescentes "xkpts.com" implementada en los centros escolares

\begin{tabular}{|c|c|c|c|c|}
\hline \multirow[t]{5}{*}{ Nivel escolar } & $\begin{array}{l}\text { Sesiones de la } \\
\text { intervención }\end{array}$ & Actividades preventivas a realizar en el aula & Técnicas a aplicar & Recursos \\
\hline & $\begin{array}{l}\text { Sesión 1: } \\
\text { "Principales conceptos } \\
\text { y contexto del con- } \\
\text { sumo de sustancias } \\
\text { adictivas" }\end{array}$ & $\begin{array}{l}6 \text { actividades en las que se plantea un debate sobre: } \\
\text { - Limites en el consumo de drogas sociales } \\
\text { - La dependencia } \\
\text { - Uso "normativo" del alcohol y mitos del alcohol } \\
\text { - Pasos en la toma de decisiones }\end{array}$ & $\begin{array}{l}\text { - Discusión } \\
\text { - Exposición en el aula } \\
\text { - Enseñanza activa* }\end{array}$ & $\begin{array}{l}\text { - Historietas del pro- } \\
\text { grama "Decideix" } \\
\text { - Guía del educador }\end{array}$ \\
\hline & $\begin{array}{l}\text { Sesión 2: } \\
\text { "Sesión didáctica audi- } \\
\text { ovisual "xkpts.com" }\end{array}$ & $\begin{array}{l}3 \text { actividades que incluyen la proyección de un DVD y posterior debate } \\
\text { sobre: } \\
\text { - El cánnabis es una droga } \\
\text { - El uso recreativo del cánnabis y quien lo consume } \\
\text { - La implicación del consumo en la edad escolar, los riesgos y los } \\
\text { efectos para la salud }\end{array}$ & $\begin{array}{l}\text { - Discusión } \\
\text { - Exposición en el aula } \\
\text { - Enseñanza activa* }\end{array}$ & $\begin{array}{l}\text { - } \text { DVD } \\
\text { - Guia didáctica de la } \\
\text { - Gesión audiovisual } \\
\text { - Guia del educador }\end{array}$ \\
\hline & $\begin{array}{l}\text { Sesión 3: } \\
\text { "Situaciones específicas } \\
\text { de consumo de cán- } \\
\text { nabis" }\end{array}$ & $\begin{array}{l}3 \text { actividades a desarrollar a partir de } 10 \text { situaciones propuestas para el } \\
\text { trabajo de las habilidades. Las situaciones se clasifican en } 3 \text { categorias: } \\
\text { - Categoría 1: Grupos con muchos no consumidores y algunos } \\
\text { experimentadores } \\
\text { - Categoria 2: Grupos con algunos no consumidores y algunos } \\
\text { experimentadores } \\
\text { - Categoría 3: Grupos con mayoria de experimentadores }\end{array}$ & $\begin{array}{l}\text { - Entrenamiento de habilidades } \\
\text { - Discusión } \\
\text { - Exposición en el aula } \\
\text { - Enseñanza activa* }\end{array}$ & - Guía del educador \\
\hline & $\begin{array}{l}\text { Sesión 4: } \\
\text { "Consecuencias del } \\
\text { consumo de sustancias } \\
\text { adictivas" }\end{array}$ & $\begin{array}{l}4 \text { actividades en las que se plantea un debate sobre: } \\
\text { - Situaciones de uso, abuso y dependencia } \\
\text { - Primeros síntomas de problemas con el alcohol } \\
\text { - Actuación ante un problema de consumo en el entorno }\end{array}$ & $\begin{array}{l}\text { - Discusión } \\
\text { - Exposición en el aula } \\
\text { - Enseñanza activa* }\end{array}$ & $\begin{array}{l}\text { - Historietas del pro- } \\
\text { grama "Decideix" } \\
\text { - Guía del educador }\end{array}$ \\
\hline Nivel familiar & & & $\begin{array}{l}\text { - Discusión sobre el tema en el } \\
\text { hogar de los jóvenes }\end{array}$ & $\begin{array}{l}\text { - Guia para las familias } \\
\text { "Cánnabis: Hablemos } \\
\text { en casa" }\end{array}$ \\
\hline $\begin{array}{l}\text { Nivel individual } \\
\text { (extraescolar) }\end{array}$ & & & $\begin{array}{l}\text { - Búsqueda de información y } \\
\text { de recursos de ayuda }\end{array}$ & $\begin{array}{l}\text { - Web del programa } \\
\text { www.xkpts.com }\end{array}$ \\
\hline
\end{tabular}

*Enseñanza activa: a partir de preguntas y respuestas 
Se recomienda la dedicación de un total de 11 horas a la aplicación de la intervención completa en el aula, incluidas las actividades principales y las opcionales. De estas 11 horas, se propone dedicar 3 horas a cada una de las 3 primeras sesiones y 2 horas para la última sesión, estableciéndose el mínimo en 1 hora para cada sesión general (sesiones 1 y 4) y 2 horas para cada sesión específica (sesiones 2 y 3 ) en caso de sólo poder realizar las actividades principales. La Figura 2 muestra el esquema de aplicación de la intervención en el aula. Todos los profesores encargados de la aplicación del programa asistieron a una jornada previa de formación en la que, además de recibir información sobre la prevención de las drogodependencias y concretamente del cánnabis, se revisaban las técnicas y recursos a utilizar durante la intervención. Posteriormente, se implementó la intervención en cada una de las 39 escuelas seleccionadas. El profesorado contaba con el soporte por parte de técnicos de la Agència de Salut Pública de Barcelona para resolver dudas sobre la implementación. Durante la aplicación del programa se distribuyó la guía de las familias y se fomentó la conexión de los escolares a la página web del programa en Internet. Asimismo, una vez se había llevado a cabo la implementación se realizó un taller de valoración del mismo en el que se discutió cómo se habian desarrollado las sesiones en las diferentes aulas y se comentaron las dificultades que habian surgido. Los profesores dedicaron un total de 8 horas a la asistencia a los dos talleres ( 6 al de formación, al inicio, y 2 al de valoración al final).

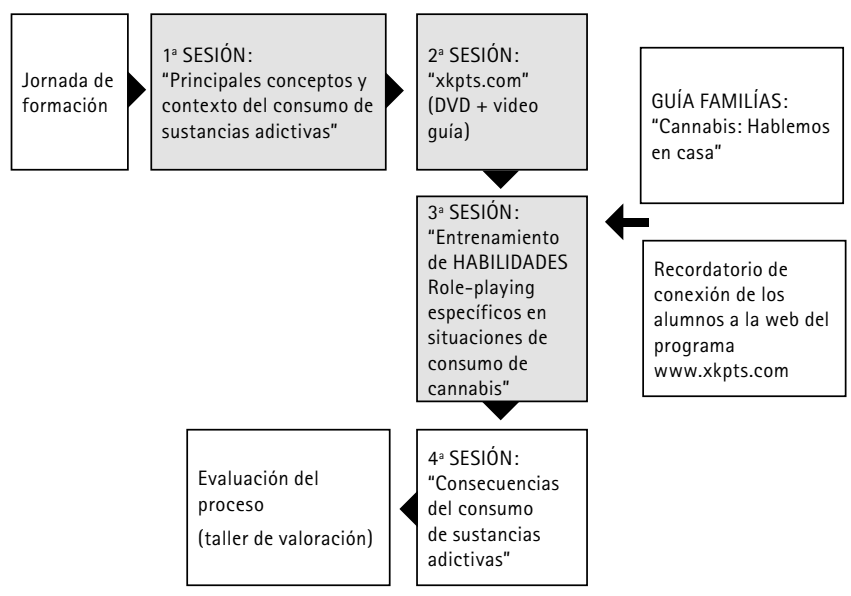

Figura 2. Esquema del programa de prevención de consumo de cánnabis en adolescentes "xkpts.com"

\section{Fuentes de información y variables estudiadas}

Para evaluar el nivel de aplicación de la intervención en las aulas, los profesores encargados de la implementación del programa en cada aula cumplimentaron una ficha de evaluación auto contestada en la que valoraron cómo se había desarrollado cada una de las 4 sesiones que componían el programa. Así pues, el método utilizado para valorar la implementación permitió medir el cumplimiento y la fidelidad autodeclarada. En esta ficha se valoraban algunos elementos importantes para juzgar la correcta aplicación del programa. Por un lado, se valoraban características relativas a la implementación de las sesiones que componen el programa, tales como el tiempo dedicado a cada una de las sesiones, las técnicas aplicadas (discusión, entrenamiento de habilidades y exposición del tema en el aula), los recursos utilizados (historietas del programa y el DVD correspondiente a la sesión didáctica audiovisual) y las actividades preventivas realizadas. Por otro, se valoraba el desarrollo de las diferentes sesiones en el aula, solicitando al profesorado que indicase el grado de acuerdo o desacuerdo con afirmaciones relativas a la atención de los alumnos, el entendimiento del contenido de la sesión por parte de los adolescentes, la facilidad para mantener la clase en calma, la satisfacción y familiaridad con los métodos educativos, así como la satisfacción con los materiales suministrados. Asimismo, con el fin de valorar cual era la satisfacción de los profesores con la intervención, se recogió tanto la puntuación que ellos otorgaban al programa, la que creian que darían los alumnos, como su intención de volver a aplicar el programa en un futuro.

Una vez finalizada la aplicación del programa, los profesores debian enviar la ficha de evaluación debidamente cumplimentada al equipo de la Agència de Salut Pública de Barcelona encargado de la dirección técnica del programa. En aquellos casos en los que no se recibió la ficha se contactó telefónicamente o vía correo electrónico con el profesorado con el fin de instarles a rellenar y enviar las fichas a la mayor brevedad posible.

Para valorar de forma global la implementación de la intervención, y de acuerdo con el buen rendimiento conseguido en un estudio previo ${ }^{30}$, se definieron operativamente dos indicadores de cumplimiento. Para ello, se tuvo en cuenta que el tiempo de que disponen las escuelas es muy variable y que las intervenciones escolares han de ofrecer un margen amplio de posibilidades para adaptarse al máximo de situaciones posibles (algunas escuelas aplican el mínimo de actividades obligatorias de una intervención mientras que en otras escuelas se trabajan casi todas las actividades propuestas). Así pues, siguiendo un criterio cuantitativo a la hora de la aplicación de las actividades en el aula, se definió como Intervención aceptable aquella en la que se habian realizado al menos 8 de las 16 actividades preventivas previstas. Por otro, siguiendo un criterio cualitativo, se definió como Intervención cualificada aquella en la que se había observado de forma selectiva la estructura de la intervención, realizando un mínimo de 1 actividad para cada sesión general y 2 actividades para cada sesión especifica.

Para valorar la participación de las familias de los adolescentes en la intervención se les envió un cuestionario solicitando que indicasen si habían recibido la guía, si la habian leído y que añadiesen los comentarios oportunos tanto sobre la intervención en el aula como sobre la guía.

Respecto a la página web, se recogió el número de visitas acumuladas hasta el 1 de julio de 2006. 
Tabla 3. Porcentaje de aulas en las que se han aplicado los diferentes componentes del programa de prevención de consumo de cánnabis en adolescentes "xkpts.com", media de actividades preventivas realizadas y tiempo medio dedicado $(n=76)$

\begin{tabular}{|c|c|c|c|c|c|c|}
\hline \multicolumn{2}{|c|}{ Componentes de la intervención } & $\begin{array}{c}\text { Sesión 1: "Principales } \\
\text { conceptos y contexto } \\
\text { del consumo de } \\
\text { sustancias adictivas" }\end{array}$ & $\begin{array}{c}\text { Sesión 2: "Sesión } \\
\text { didáctica audiovisual } \\
\text { "xkpts.com" }\end{array}$ & $\begin{array}{c}\text { Sesión 3: "Situaciones } \\
\text { específicas de consumo } \\
\text { de cánnabis" }\end{array}$ & $\begin{array}{l}\text { Sesión 4: "Consecuencias } \\
\text { del consumo de } \\
\text { sustancias adictivas" }\end{array}$ & Total \\
\hline & & $(\%)$ & $(\%)$ & $(\%)$ & $(\%)$ & $(\%)$ \\
\hline \multirow{4}{*}{ Técnicas aplicadas } & Discusión & $89,5 \%$ & $72,4 \%$ & $64,5 \%$ & $53,9 \%$ & $100 \% \%^{+}$ \\
\hline & $\begin{array}{l}\text { Entrenamiento de habili- } \\
\text { dades }\end{array}$ & $27,6 \%$ & $5,3 \%$ & $59,2 \%{ }^{*}$ & $9,2 \%$ & $72,4 \% 0^{+}$ \\
\hline & Exposición & $57,9 \%$ & $36,8 \%$ & $31,6 \%$ & $32,9 \%$ & $72,4 \% \%^{+}$ \\
\hline & $\begin{array}{l}\text { Enseñanza activa ( a partir } \\
\text { de preguntas y respuestas) }\end{array}$ & $23,7 \%$ & $6,6 \%$ & $7,9 \%$ & $10,5 \%$ & $27,6 \% 0^{+}$ \\
\hline \multirow{2}{*}{ Recursos } & Historietas & $82,9 \% \%^{+}$ & $18,4 \%$ & $38,2 \%$ & $60,5 \% \%^{*}$ & $86,8 \% \%^{+}$ \\
\hline & DVD & $38,2 \%$ & $84,2 \%^{+}$ & $15,8 \%$ & $14,5 \%$ & $93,4 \% 0^{+}$ \\
\hline \multirow[b]{2}{*}{$\begin{array}{l}\text { Actividades } \\
\text { preventivas }\end{array}$} & $\begin{array}{l}\text { Media de actividades reali- } \\
\text { zadas }\end{array}$ & 3,12 de 6 & 2,24 de 3 & 2,20 de 3 & 1,66 de 4 & 9,22 de 16 \\
\hline & $\begin{array}{l}\text { Actividad realizada con más } \\
\text { frecuencia por el profesorado }\end{array}$ & $\begin{array}{l}\text { Discusión grupal sobre } \\
\text { una historieta }(77,6 \%)\end{array}$ & $\begin{array}{l}\text { Visión del DVD y dis- } \\
\text { cusión }(84,2 \%)\end{array}$ & $\begin{array}{l}\text { Una de las situaciones de } \\
\text { la Categoria } 1^{*}(71,1 \%)\end{array}$ & $\begin{array}{l}\text { Discusión grupal sobre una } \\
\text { historieta }(57,9 \%)\end{array}$ & $\begin{array}{l}\text { Visión del } \\
\text { DVD y } \\
\text { discusión } \\
(84,2 \%)\end{array}$ \\
\hline \multicolumn{2}{|c|}{ Tiempo medio dedicado } & $1 \mathrm{~h} 35 \mathrm{~min}$ & 1h $35 \mathrm{~min}$ & $1 \mathrm{~h} 15 \mathrm{~min}$ & 1h $15 \mathrm{~min}$ & $5 \mathrm{~h} 40 \mathrm{~min}$ \\
\hline
\end{tabular}

${ }^{+}$Realizado en alguna de las 4 sesiones

${ }^{\dagger}$ Realizado en la sesión indicada en el protocolo del programa

*Categoría 1: grupos con muchos no consumidores y algunos experimentadores

\section{Análisis estadístico}

Se realizó un análisis descriptivo de las variables incluidas en el estudio. En el caso de las variables categóricas se calcularon las frecuencias (absolutas y relativas), mientras que para las variables cuantitativas se calcularon medidas de tendencia central (media, mediana, mínimo y máximo). Para el procesamiento y análisis de los datos se utilizó el paquete estadístico SPSS 12.0.

\section{RESULTADOS}

De las 117 aulas en las que se aplicó el programa, se recibió la ficha de evaluación de los profesores de 76 de ellas, siendo la tasa de respuesta del 65\%. Las 76 aulas de las que se recibió la ficha pertenecian a 32 de las 39 escuelas participantes en el estudio.

El tiempo medio dedicado a la aplicación de la intervención en las aulas fue de 5 horas y 40 minutos, disminuyendo el tiempo dedicado a cada sesión a medida que se avanzaba en el programa. De las diversas técnicas propuestas en el protocolo del programa para el desarrollo de las sesiones, fue la discusión la que se aplicó con mayor frecuencia, ya que en el $100 \%$ de las aulas se discutió sobre el tema en algún momento de la intervención. El entrenamiento de habilidades y la exposición del tema en el aula se aplicaron cada una en el $72,4 \%$ de los casos. El entrenamiento de habilidades fue aplicado en un 59,2\% de las aulas en la tercera sesión tal y como se especificaba en el protocolo mientras que un 13,2\% lo aplicaron en una sesión distinta a la indicada. De los recursos facilitados, el DVD fue el más utilizado ya que se proyectó en el 93,4\% de las aulas aunque el 9,2\% de ellas lo trabajaron en una sesión diferente a la segunda, que era la indicada según el protocolo. En cuanto a las actividades preventivas, la media de actividades realizadas fue de 9,22 de las 16 propuestas, siendo en la segunda y en la tercera sesión donde se realizó una mayor proporción de actividades, 2,24 de 3 y 2,20 de 3 respectivamente. Del total de actividades propuestas, la visión del DVD y una posterior discusión fue la realizada con mayor frecuencia, en un $84,2 \%$ de las aulas (Tabla 3 ).

La Tabla 4 muestra cuál es la percepción de los profesores sobre el funcionamiento del programa en el aula. El cálculo para cada una de las sesiones ha sido realizado sobre el total de aulas de las que se obtuvo respuesta a las cuestiones planteadas para esa sesión. Se aprecia una disminución de la tasa de respuesta a medida que avanzaban las sesiones, siendo del 97,4\% para las sesiones 1 y 2, del 81,6\% para la sesión 3 y del 67,1\% para la última. Los profesores indicaron que los alumnos comprendieron correctamente el contenido de los temas tratados y que les fue fácil mantener la clase en calma en el 100\% de las aulas en las que se aplicó el programa. Los alumnos estuvieron atentos en el 98,7\% de 
Tabla 4. Percepción del profesorado sobre el funcionamiento del programa de prevención de consumo de cánnabis en adolescentes "xkpts.com" en aquellas aulas que han respondido la ficha de evaluación (total de aulas $=76$ )

\begin{tabular}{|c|c|c|c|c|c|}
\hline $\begin{array}{l}\text { Percepción del profesorado del fun- } \\
\text { cionamiento del programa }\end{array}$ & $\begin{array}{c}\text { Sesión 1: "Principales } \\
\text { conceptos y contexto del } \\
\text { consumo de sustancias } \\
\text { adictivas" }\end{array}$ & $\begin{array}{c}\text { Sesión 2: } \\
\text { "Sesión didáctica audiovisual } \\
\text { "xkpts.com" }\end{array}$ & $\begin{array}{c}\text { Sesión 3: "Situaciones } \\
\text { especificas de consumo de } \\
\text { cánnabis" }\end{array}$ & $\begin{array}{c}\text { Sesión 4: } \\
\text { "Consecuencias del consumo } \\
\text { de sustancias adictivas" }\end{array}$ & Total $^{*}$ \\
\hline & n (\%) & $n(\%)$ & $n(\%)$ & n (\%) & n (\%) \\
\hline Porcentaje de respuesta obtenida & $74(97,4 \%)$ & $74(97,4 \%)$ & $62(81,6 \%)$ & $51(67,1 \%)$ & $74(97,4 \%)$ \\
\hline Los alumnos están atentos & $91,9 \%$ & $98,7 \%$ & $95,0 \%$ & $92,2 \%$ & $98,7 \%$ \\
\hline $\begin{array}{c}\text { Los alumnos comprenden el conte- } \\
\text { nido de la sesión }\end{array}$ & $94,6 \%$ & $99,9 \%$ & $98,3 \%$ & $92,1 \%$ & $100 \%$ \\
\hline $\begin{array}{l}\text { El profesor mantiene la clase en } \\
\text { calma con facilidad }\end{array}$ & $85,1 \%$ & $87,9 \%$ & $90,3 \%$ & $94,0 \%$ & $100 \%$ \\
\hline $\begin{array}{c}\text { El profesor conoce las técnicas } \\
\text { educativas }\end{array}$ & $95,8 \%$ & $95,9 \%$ & $98,3 \%$ & $98,1 \%$ & $97,3 \%$ \\
\hline $\begin{array}{l}\text { El profesor está satisfecho con las } \\
\text { técnicas educativas }\end{array}$ & $90,5 \%$ & $91,8 \%$ & $96,8 \%$ & $96,0 \%$ & $97,3 \%$ \\
\hline $\begin{array}{l}\text { El profesor está satisfecho con los } \\
\text { recursos suministrados }\end{array}$ & $95,9 \%$ & $95,9 \%$ & $93,6 \%$ & $100 \%$ & $93,2 \%$ \\
\hline
\end{tabular}

El cálculo para cada sesión se realiza sobre el número de aulas en que se responden estas cuestiones

"Si observamos el indicador en 2 o más sesiones

Tabla 5. Clasificación de las aulas según el tipo de implementación del programa "xkpts.com" y satisfacción del profesorado con el mismo $(n=76)$

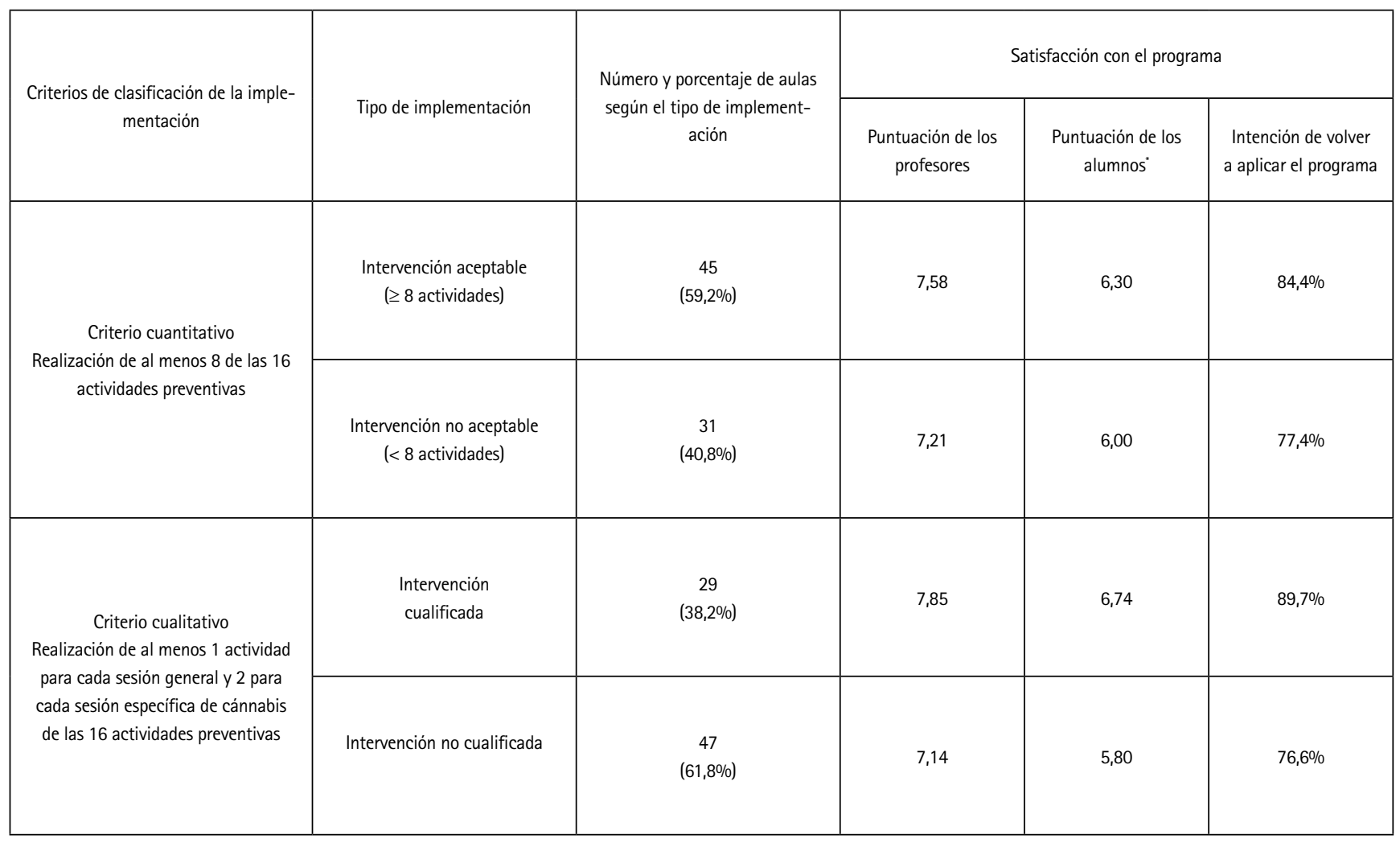

*Puntuación que los profesores creen que sus alumnos otorgarian al programa 
las aulas. Por otro lado, el profesorado indicó que conocía los métodos educativos y estaba satisfecho con ellos en el $97,3 \%$ de las aulas y que estaba satisfecho con los recursos suministrados en el 93,2\%.

En la Tabla 5 podemos observar el número y el porcentaje de aulas en las que se realizó la intervención en función de los dos criterios de implementación establecidos, cuantitativo y cualitativo. El número de aulas en las que, siguiendo el criterio cuantitativo, se realizó una intervención aceptable fue mayor que el de aulas en las que, según el criterio cualitativo, se realizó una intervención cualificada $(59,2 \%$ vs. $38,2 \%$ ). Los profesores otorgaron una puntuación media de 7,42 sobre 10 al programa, mostrando el $81,6 \%$ de ellos intención de volver a aplicarlo en un futuro. Los resultados muestran un gradiente en la satisfacción con el programa en función del tipo de indice de cumplimiento obtenido. La mayor satisfacción se observa en las aulas en las que la intervención fue cualificada, así como la mayor puntuación otorgada al programa tanto por parte del profesorado $(7,85)$ como la estimada que daría el alumnado $(6,74)$. Asimismo, es en las aulas con una intervención cualificada en las que un mayor porcentaje de profesores indica que volvería a aplicar la intervención (89,7\%). Los profesores menos satisfechos con el programa son aquellos que realizaron una intervención no cualificada.

El 28,3\% (796) de las familias cumplimentó el cuestionario sobre la guía enviada a los domicilios de los escolares. De éstas, el 69,8\% (556) refería haber leído la guía, el 23,6\% (188) haberla recibido pero todavía no haberla leído y el 6,5\% (52) no haberla recibido.

Los cuestionarios recibidos correspondían a 74 de las 117 aulas intervenidas, siendo la tasa de respuesta por aula del $63,3 \%$. Para cada una de las aulas, el número de familias que envió el cuestionario cumplimentado fue muy variable, siendo la media de 11 familias (mínimo 3 y máximo 30). Del $87,8 \%$ de las aulas de las que se recibió el cuestionario de las familias también se habia recibido la ficha de evaluación de los profesores $y$, en todas ellas, la intervención fue aceptable y/o cualificada $(62,2 \%$ aulas con intervención aceptable y $67,6 \%$ con intervención cualificada).

Por otro lado, el número de visitas acumuladas a la página web del programa hasta el 1 de julio de 2006 fue de 2731.

\section{DISCUSIÓN}

Nuestro estudio aporta información detallada sobre la evaluación de proceso del programa de prevención del consumo de cánnabis "xkpts.com" en el entorno escolar, mostrando una clara relación entre el tipo de implementación aplicada en el aula y la valoración que el profesorado hace del programa. Aquellos profesores que han realizado una intervención cualificada son los que otorgan la mayor puntuación al programa y los que expresan mayor intención de volver a aplicarlo en el futuro. Nuestros resultados muestran que a mayor cumplimiento de la implementación mayor es la satisfacción del profesorado con la intervención. Sin embargo, tan sólo en dos de cada cinco aulas se realizó una intervención cualificada, dato que concuerda con la evidencia científica que muestra la dificultad de conseguir una implementación adecuada en las intervenciones preventivas escolares ${ }^{10,14-16}$. En el caso del criterio cuantitativo de implementación, que evalúa la intervención en función del número de actividades realizadas, la intervención fue correcta en tres de cada cinco aulas. Así pues, como podemos observar, el cumplimiento por parte de los profesores en cuanto al número total de actividades realizadas en la aplicación de las intervenciones preventivas es mejor que el seguimiento del protocolo del programa, es decir, que la fidelidad a las recomendaciones sobre la forma de realizarlas.

Cabe destacar que la gran mayoría de los profesores que aplicaron el programa mostraron satisfacción con los materiales suministrados. Asimismo, prácticamente en todas las aulas se utilizó el DVD correspondiente a la sesión didáctica audiovisual como recurso principal del programa, dato que corrobora la afirmación de otros autores de que disponer de materiales bien preparados y estructurados facilita la implementación ${ }^{15}$. De hecho, algunos estudios señalan que disponer de unos materiales claros y explícitos es una de las características más importantes de los programas de prevención escolar para conseguir una implementación de calidad. Esto permite al profesorado centrar su atención en la enseñanza, ahorrar tiempo, facilita la implementación y minimiza el riesgo de desviación del contenido de las sesiones $^{15,31}$.

Por otro lado, el entrenamiento de habilidades ha sido una técnica realizada en tres de cada cuatro aulas en las que se aplicó la intervención. Este resultado representa un éxito en el desarrollo de la implementación del programa ya que la literatura científica ha evidenciado que los programas preventivos escolares que incorporan contenidos que ayudan a identificar la presión social para consumir y promueven las habilidades para rechazar su consumo son los que permiten alcanzar mayor efectividad en la prevención del consumo de sustancias entre los jóvenes ${ }^{1,32-37}$.

Nuestros resultados sugieren que la participación de las familias de los adolescentes está muy ligada al tipo de intervención realizada en el aula. Así pues, en 9 de cada 10 aulas de las que se recibieron los cuestionarios de las familias la intervención fue aceptable y/o cualificada. Estos datos apuntan que, posiblemente, los docentes más comprometidos con la adecuada implementación de la intervención en el aula también ponen un mayor interés en hacer a las familias partícipes en el proyecto.

Algunos estudios han señalado la utilidad de la evaluación de proceso como fuente de información ya que permite evaluar la implementación realizada, mejorar el programa en base a las sugerencias realizadas y ayudar a otros profesionales a conducir intervenciones similares ${ }^{20,38}$. De nuestra experiencia se desprende que el cuestionario auto contestado por el profesorado es una herramienta de gran utilidad para medir la implementación y que permite medir tanto el cumplimiento como la fidelidad autodeclarada 
de la implementación. Sin embargo, una limitación del cuestionario auto contestado como método de medición de la implementación es el posible sesgo de información debido al deseo de agradar en las respuestas, con la consiguiente sobreestimación de la aplicación del programa. A su vez, el hecho de que los cuestionarios fuesen cumplimentados una vez finalizada la intervención, en lugar de hacerlo inmediatamente después de cada sesión, podría afectar a la precisión y veracidad de las respuestas. Entre las posibles estrategias para mejorar la validez de este método se incluye estimular al profesorado mediante incentivos para que cumplimenten el formulario, asegurándole que en ningún caso habrá consecuencias negativas en caso de declarar una escasa implementación.

Otra limitación de nuestro estudio radica en utilizar únicamente un método de medición de la implementación en el aula. Así pues, consideramos que sería de gran utilidad complementar el uso de cuestionarios auto contestados con otros métodos, tales como la observación no participante en el aula y la entrevista post-implementación al profesor ${ }^{11}$.

Asimismo, el hecho de que las escuelas participantes en el estudio ya estuviesen aplicando un programa de prevención de drogodependencias podría ser un sesgo de selección ya que, a priori, se podría entender que se trata de escuelas que están más sensibilizadas con el problema del consumo de drogas en la adolescencia $y$, por tanto, más comprometidas a la hora de aplicar correctamente la intervención. No obstante, consideramos que una de las fortalezas del estudio es el tamaño de muestra, con la participación de 39 escuelas, muestra muy superior a la de otros estudios $11,14,16,17,39,40$.

Diversos estudios muestran que las intervenciones con menor calidad de implementación son las que conducen a resultados de efectividad más pobres ${ }^{3,4,12,41}$. Un estudio realizado por Domitrovich y Greenberg (2000) mostró que solo el $21 \%$ de 34 programas preventivos efectivos estudiados examinaba si la calidad de la implementación se relacionaba con los resultados obtenidos ${ }^{42}$.

El éxito de una intervención preventiva depende en gran medida de la fidelidad de la implementación. La correcta planificación de la evaluación de proceso es fundamental para obtener indicadores válidos que, en la evaluación de la efectividad de la intervención, nos permitan determinar qué estrategias han sido las más eficaces en la obtención de unos resultados positivos. Nuestro estudio, atendiendo a la literatura científica sobre el tema, es uno de los pocos en nuestro medio que aporta información sobre el desarrollo preciso de la intervención. Asimismo, proporciona orientaciones sobre cómo planificar una adecuada evaluación de proceso y recomendaciones para optimizarla, ya que en nuestro entorno en pocas ocasiones se evalúa la aplicación de las intervenciones escolares $y$, cuando se hace, suelen ser de manera poco rigurosa e incompleta.

Finalmente, al tratarse de un estudio que se engloba en un proyecto más amplio de evaluación de la efectividad del programa de prevención de consumo de cánnabis "xkpts. com", los resultados de la evaluación de proceso nos permitirán comprobar en un futuro cercano si los resultados obtenidos sobre la conducta de los escolares se pueden relacionar con la correcta implementación del programa.

\section{AGRADECIMIENTOS}

Este estudio ha sido parcialmente financiado por el Plan Nacional Sobre Drogas (2005) y la Fundación Privada Caixa Catalunya (Área de Atención Social). Los autores quieren agradecer la participación de los centros escolares incluidos en el estudio, así como la colaboración en la recogida de información del personal del Servei de Salut Comunitària de la Agència de Salut Pública de Barcelona.

\section{REFERENCIAS}

1. Tobler NS. Drug prevention programs can work: Research findings. J Addict Dis. 1992;11:1-28.

2. Ennett ST, Tobler NS, Ringwalt $C L$, Flewelling RL. How effective is drug abuse resistance education? A meta-analyses of Project DARE outcome evaluations. Am J Public Health. 1994;84:13941404.

3. Botvin GJ, Baker E, Dusenbury L, Botvin EM, Diaz T. Long-term follow-up results of a randomized drug abuse prevention trial in a white middle-class population. JAMA. 1995;273:1106-1112.

4. Botvin GJ, Schinke S, Orlandi MA. School-based health promotion: Substance abuse and sexual behavior. Appl Prev Psychol. 1995;4:167-184.

5. Wiehe SE, Garrison MM, Christakis DA, Ebel BE, Rivara FP. A systematic review of school-based smoking prevention trials with long-term follow-up. J Adolesc Health. 2005 Mar;36:162169.

6. Klepp K, Tell GS, Vellar OD. Ten-year follow-up of the Oslo Youth Study smoking prevention program. Prev Med. 1993;22:453462.

7. Bell RM, Ellickson PL, Harrison ER. Do drug prevention effects persist into high school? How Project Alert did with ninth graders. Prev Med. 1993;22:463-483.

8. Flay BR, Phil D, Koepke D, Thomson SJ, Santi S, Best A, et al. Sixyear follow-up of the first Waterloo school smoking prevention trial. Am J Public Health. 1989;79:1371-1376.

9. Murray DM, Pirie $P$, Luepker RV, Pallonen U. Five and six-year follow-up results from four seventh-grade smoking prevention strategies. J Behav Med. 1989;12:207-218.

10. Resnicow K, Botvin G. School-based substance use prevention programs: Why do Effects decay? Prev Med. 1993;22:484-490.

11. Resnicow $K$, Davis M, Smith M, Lazarus-Yaroch A, Baranowski $T$, Baranowski J, et al. How best to measure implementation of school health curricula: a comparison of three measures. Health Educ Res. 1998;13:239-250.

12. Dusenbury $L$, Branningan $R$, Falco $M$, Hansen WB. A review of research on fidelity of implementation: implications for drug abuse prevention in school settings. Health Educ Res. 2003;18:237-56.

13. Orwin RG. Assessing program fidelity in substance abuse health services research. Addiction. 2000;95:309-327.

14. Dusenbury L, Brannigan R, Hansen WB, Walsh J, Falco M. Quality of implementation: developing measures crucial to 
understanding the diffusion of preventive interventions. Health Educ Res. 2005 Jun;20:308-313.

15. Payne AA, Gottfredson DC, Gottfredson GD. School predictors of the intensity of implementation of school-based prevention programs: results from a national study. Prev Sci. 2006 Jun;7:225-37.

16. Pankratz MM, Jackson-Newsom J, Giles SM, Ringwalt $C L$, Bliss $\mathrm{K}$, Bell ML. Implementation fidelity in a teacher-led alcohol use prevention curriculum. J Drug Educ. 2006;36:317-333.

17. Sánchez $V$, Steckler $A$, Nitriat $P$, Hallfors $D, C h o ~ H$, Brodish $P$. Fidelity of implementation in a treatment effectiveness trial of Reconnecting Youth. Health Educ Res. 2007;22:95-107.

18. Rohrbach LA, Dent CW, Skara S, Sun P, Sussman S. Fidelity of Implementation in Project Towards No Drug Abuse (TND): A comparison of classroom teachers and program specialists. Prev Sci. 2007;8:125-132.

19. Resnicow K, Cross D, Wynder E. The Know your body program: a review of evaluation studies. Bull N Y Acad Med. 1993 Winter;70:188-207.

20. McGrath Y, Sumnall H, Edmonds K, McVeigh J, Bellis M. Review of grey literature on drug prevention among young people. London: National Institute for Health and Clinical Excellence. May 2006

21. Soldano C, Markell G. Parent involvement in health concerns for youth: the issue of adolescent immunization. J Sch Health. 1997 Sep;67:292-3.

22. Calafat A, Amengual M. Educación sobre el alcohol. Madrid: Delegación del Gobierno para el Plan Nacional sobre Drogas, Ministerio del Interior; 1999.

23. Henricson $C_{1}$ Roker D. Support for the parents of adolescents: a review. J Adolesc. 2000 Dec;23:763-83. Review.

24. Sanders MR. Community-based parenting and family support interventions and the prevention of drug abuse. Addict Behav. 2000 Nov-Dec;25:929-42.

25. Al-Halabí Díaz S, Errasti Pérez JM, Fernández Hermida JR. El colegio y los factores de riesgo familiar en la asistencia a programas de prevención familiar del consumo de drogas. Adicciones. 2009;21:39-48.

26. Pérez JM, Díaz SA, Fernández Hermida JR, Villa RS, Carballo Crespo JL, Rodríguez OG. Recruitment characteristics influencing parental participation in family-based drug-abuse prevention programs: the Spot and Redmond model in Spain. Subst Use Misuse. 2008;43:850-7.

27. Ariza $C$, Pérez $A$, Nebot $M$, Juárez 0 , Rodríguez-Martos $A$. Programa de prevención del consumo de cánnabis "xkpts.com" (protocolo, DVD y guia del educador). Agencia de Salud Pública de Barcelona. Ed. Fundación Viure i Conviure de la Obra Social de Caixa Catalunya. Barcelona; 2006.

28. Calafat $A$, Amengual $M$, Guimerans $C$, Rodriguez-Martos $A$, Ruiz R. "Tú decides": 10 años de un programa de prevención escolar. Adicciones. 1995;7:509-526.

29. Ariza C, Rodríguez-Martos A, Vecino C, Guitart A. Cannabis: Hablemos en casa. Agencia de Salud Pública de Barcelona. Ed. Fundación Viure i Conviure de la Obra Social de Caixa Catalunya. Barcelona, 2006

30. Ariza $C$, Nebot $M$, Tomás $Z$, Giménez $E$, Valmayor $S$, Tarilonte $V$, et al. Longitudinal effects of the European smoking prevention framework approach (ESFA) project in Spanish adolescents. Eur J Public Health. 2008 Jun 18. (Epub ahead of print).
31. Kealey KA, Peterson AV Jr, Gaul MA, Dinh KT. Teacher training as a behavior change process: principles and results from a longitudinal study. Health Educ Behav. 2000 Feb;27:64-81.

32. Tobler NS. Meta-analysis of 143 adolescent drug prevention programs: Quantitative outcome results of program participants compared to a control or comparison group. J Drug Issues. 1986;16:537-567.

33. Tobler NS. Meta-analysis of adolescent drug prevention programs: Results of the 1993 meta-analysis. NIDA Res monogr. 1997;170:5-68.

34. Tobler NS, Stratton HH. Effectiveness of school-based drug prevention programs: A meta-analysis of the research. J Prim Prev. 1997;18:71-128.

35. Tobler NS, Lessard $T$, Marshall D, Ochshorn $P$, Roona M Effectiveness of school-based drug prevention programs for marijuana use. Sch Psychol Int. 1999;20:105-137.

36. Tobler NS, Roona MR, Ochshorn P, Marshall DG, Streke AV, Stackpole KM. School-based adolescent drug prevention programs: 1998 meta-analysis. J Prim Prev. 2000;20:275-336.

37. Dusenbury L, Falco M. Eleven components of effective drug abuse prevention curricula. J Sch Health. 1995 Dec;65:420-5.

38. WHO, UNDCP \& EMCDDA. Evaluation of psychoactive substance use disorder treatment, workbook 4: process evaluations. 2000. Geneva: World Health Organization.

39. Rohrbach L, Graham J, Hansen W. Diffusion of a school-based substance abuse prevention program: predictors of program implementation. Prev Med. 1993; 22:237-260.

40. Kam CM, Greenberg MT, Walls CT. Examining the role of implementation quality in school-based prevention using the PATHS curriculum. Prev Sci. 2003;4:55-63.

41. Battistich V, Schaps E, Watson M, Solomon D. Prevention effects of the child development project: Early findings from an ongoing multi-site demonstration trial. J Adolesc Res. 1996;11:12-35.

42. Domitrovich CE, Greenberg MT. The study of implementation: Current findings from effective programs that prevent mental disorders in school-aged children. J Educ Psychol Consult. 2000;11:193-221. 